\title{
Wireless Multi-group Multicast Precoding with Selective RF Energy Harvesting
}

\author{
Sumit Gautam, Eva Lagunas, Symeon Chatzinotas and Björn Ottersten \\ Interdisciplinary Centre for Security, Reliability and Trust (SnT), University of Luxembourg, Luxembourg. \\ Email: \{sumit.gautam,eva.lagunas,symeon.chatzinotas,bjorn.ottersten\}@uni.lu
}

\begin{abstract}
We present a novel framework for multi-group multicast precoding in the presence of three types of wireless users which are distributed among various multicast groups. A multi-antenna transmitter conveys information and/or energy to the groups of corresponding receivers using more than one multicast streams. The information specific users have conventional receiver architectures to process data, energy harvesting users collect energy using the non-linear energy harvesting module and each of the joint information decoding and energy harvesting capable user is assumed to employ the separated architecture with disparate non-linear energy harvesting and conventional information decoding units. In this context, we formulate and analyze the problem of total transmit power minimization for optimal precoder design subjected to minimum signal-tointerference-and-noise ratio and harvested energy demands at the respective users under three different scenarios. This problem is solved via semi-definite relaxation and the advantages of employing separate information and energy precoders are shown over joint and per-user information and energy precoder designs. Simulation results illustrate the benefits of proposed framework under several operating conditions and parameter values.
\end{abstract}

\section{INTRODUCTION}

The current and future generation of wireless communication devices raise several challenges like increasing capacity and performance needs, complex hardware circuitry, and demands for energy-efficient algorithms. Additionally, it is needless to mention that the limited battery sources act as the backbone of these wireless devices to tackle their assertive demands, which is continuously weakened due to the rapid battery drainage. In this regard, optimization of power consumption helps in improving the battery duration on the one hand while energy harvesting techniques comes in handy as a recharging alternative on the other [1], [2]. Moreover, it is crucial to ensure that various types of devices like information decoding (ID) specific, explicit to energy harvesting (EH), and the ones performing ID and EH simultaneously, co-exist within the wireless networks and reap maximum benefits.

Implementation of Multiple-Input Single-Output (MISO) systems is shown to be beneficial for information specific users [3]. The notion of joint transmission of radio-frequency (RF) information and energy was proposed in [4] and further extended to multi-user MISO case in [1], [5]. In this context, several receiver architectures like time-switching (TS), powersplitting (PS), separated architecture (SA) and integrated architecture (IA) are proposed to perform ID and EH simultaneously [6]. Noticeably, the TS, PS, and integrated receiver architectures involve complex circuitries with additional opti- mization parameter(s) to distribute the received signal for ID and $\mathrm{EH}$ purposes either distinctly or concurrently (over a time period), in comparison to SA.

In order to increase the channel capacity and diversity in multi-user MISO systems, transmit precoding shows great promise [7], [8]. Multi-group (MG) Multicast (MC) is another potential technique to significantly improve the system performance. The benefits of MG-MC precoding are demonstrated in [9], [10]. However, it was shown in [7] that an $\mathrm{MC}$ precoding problem is NP-hard by nature even for single group multicasting. Several works discuss joint information and energy transfer in the MG-MC scenario [11]-[13], however, with an assumption of a linear EH module. A framework to investigate PS-based SWIPT Multicasting was presented in [14] with individual QoS constraints to meet the demands of energy sustainable Internet-of-Things (IoT). It is noteworthy that these works do not consider coexistence of different user types in the case of MG-MC.

In this paper, we consider the problem of MG-MC precoding to minimize the overall transmit power of a MISO system. The transmit source is assumed to be equipped with an array of antennas which serves multiple users with the aid of beamforming through adequate precoders. In this context, we investigate this problem in three different scenarios, considering the aspect of co-existence of three different types of users. The non-linear EH constraint and the non-convex objective of the aforementioned problem lead to intractability. We propose adequate transformation of the non-linear $\mathrm{EH}$ constraint to a linear one, and make the problem convex with the help of semi-definite relaxation (SDR). Specifically, the main contributions and novelty of this work are listed below

(a) We consider a novel MG-MC precoding framework which deals with co-existence of three types of users capable of information decoding, energy harvesting, and joint information and energy extraction, respectively.

(b) We provide adequate transformation to reduce the nonlinear EH constraint to a linear form. Without loss of generality, this transformation may come in handy to solve similar problems with non-linear EH constraints.

(c) We show that separate information and/or energy precoder design has superior performance benefits over joint or per-user information and/or energy precoder designs.

Further sections of this paper are organized as follows. Section II provides an introduction to the system model. The problem formulation and the proposed solution are presented 
27th European Signal Processing Conference (EUSIPCO’19), 2-6 September 2019, A Coruña, Spain

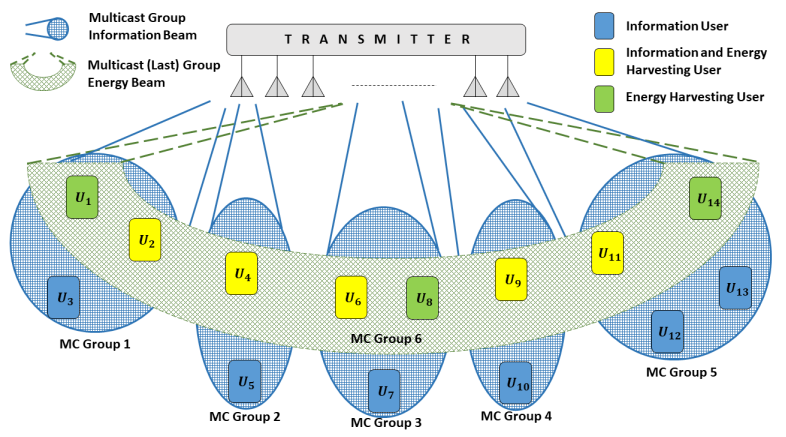

Fig. 1: System model for Separate Multicast and Energy Precoding Design (SMEP).

in Section III. Numerical results are shown in Section IV, followed by concluding remarks in Section V.

\section{SYSTEM MODEL}

The system set-up considered in this paper comprises of a transmitter equipped with $M$ antennas that provides coverage to $K$ users $\left(\mathcal{U}_{1}, \ldots, \mathcal{U}_{K}\right)$. We distinguish three types of users, namely, users able to decode information, users able to harvest energy, and users capable of doing both, respectively. The latter are assumed to be equipped with two separate RF chains designed exclusively to carry out the desired operations of ID and EH. This design is often referred in the literature as the separated architecture (SA) for enabling joint information processing and energy harvesting [6] at the receiver.

We propose and analyze three different precoding design approaches for transmit power minimization, which are discussed below

(a) Separate Multicast and Energy Precoding Design (SMEP): In this case, we assume $Z$ multicast information groups and an additional group devoted to $\mathrm{EH}$ specific users. Thus, we target design of atleast $(Z+1)$ precoders.

(b) Joint Multicast and Energy Precoding Design (JMEP): Herein, no exclusive precoder is present for EH specific users. In particular, we target the design of $Z$ multicast precoders taking into account the information and/or energy demands of the corresponding users.

(c) Per-User Information and/or Energy Precoding Design (PIEP): We assume in this case that each user is served by a single dedicated precoder. Therefore, we target the design of $K$ precoders (equal to the number of users).

For illustration purpose, we consider an example with $K$ $=14$ users $(6$ users are ID specific, 3 users only harvest energy, and 5 users have joint ID and EH capabilities), $M$ transmit antennas (with $M \geq K$ for PIEP to work), and $Z$ $=5 \mathrm{MC}$ groups. Correspondingly, the system set-up for the aforementioned three scenarios (SMEP, JMEP, and PIEP) are depicted in Fig. 1, Fig. 2, and Fig. 3, respectively. Let $\mathcal{Z}_{k}$ denote the $k^{\text {th }}$ multicast group of users. We assume known MC groups and the last EH group in this work ${ }^{1}$. Let us also

\footnotetext{
${ }^{1}$ In JMEP, it is clear that some EH users from EH specific group are also present in various $Z$ MC groups. Regarding categorization of (remaining) EH users within the $Z$ groups, certain methods e.g., distance from the nearest transmit antenna, distance from the nearest beam, etc., may be applied. This, however, involves rigorous analysis and is out of the scope of this work.
}

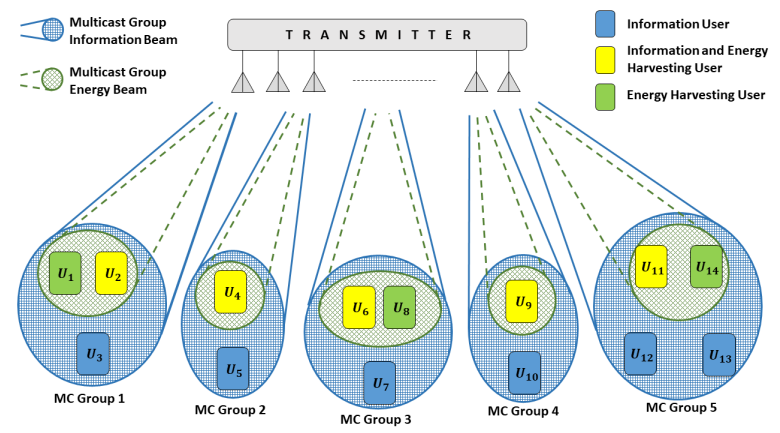

Fig. 2: System model for Joint Multicast and Energy Precoding Design (JMEP).

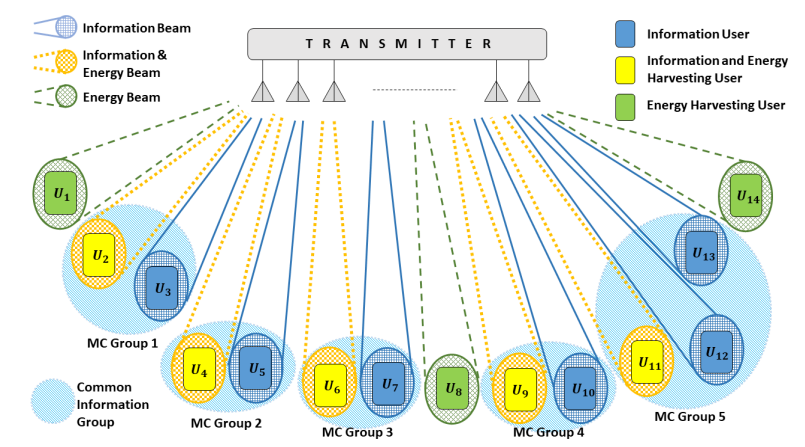

Fig. 3: System model for Per-User Information and/or Energy Precoding Design (PIEP).

define the following variable to assist the precoding design metrics in three cases interchangeably

$$
\Psi=\left\{\begin{array}{cll}
Z+1 & : & \text { SMEP } \rightarrow \psi(a) . \\
Z & : & \text { JMEP } \rightarrow \psi(b) . \\
K & : & \text { PIEP } \rightarrow \psi(c) .
\end{array}\right.
$$

Each user adheres to listen only the $\mathrm{MC}$ specific for the group where corresponding user is present i.e., $\mathcal{Z}_{k} \cap \mathcal{Z}_{\ell}=\varnothing$, $\forall k, \ell=\{1, \ldots, \Psi\}$ and $k \neq \ell$; whereas in case of $\mathrm{EH}$, the user harvests energy using all the possible multicast signals ${ }^{2}$.

The antenna array at the transmitter emits the signal $\mathbf{x}(t)=$ $\sum_{k=1}^{\Psi} \mathbf{w}_{k} a_{k}(t)$, where $\mathbf{w}_{k}$ is the related $M \times 1$ complex precoding weight vector for the users in group $\mathcal{Z}_{k}$, and $a_{k}(t)$ is the corresponding information and/or energy signal. Additionally, we assume that the information and/or energy signals for each group $\left\{a_{k}(t)\right\}_{k=1}^{\Psi}$ are mutually uncorrelated to each other with zero mean and unit variance, $\sigma_{a_{k}}^{2}=1$. The corresponding ID and/or EH signals may be separately designed according to the framework proposed in [15]. Distinct ID and EH signal forms motivate the use of SA-based devices. The total transmitted power can thus be given by $\sum_{k=1}^{\Psi} \mathbf{w}_{k}^{H} \mathbf{w}_{k}$.

The received signal at the $i^{\text {th }}$ user is given by $y_{i}(t)=$ $\mathbf{g}_{i}^{H} \mathbf{x}(t)+n_{R, i}(t)$, where $\mathbf{g}_{i}$ is the $M \times 1$ conjugated channel vector for the corresponding receiver and $n_{R, i}(t)$ is the additive zero mean Gaussian noise at the corresponding $i^{\text {th }}$ user's receiving antenna equipment with a noise variance of $\sigma_{R, i}^{2}$. The source signals are uncorrelated with $n_{R, i}(t)$. The

\footnotetext{
${ }^{2}$ The other MCs are primarily taken into consideration due to interference causing side-lobes other than the desired MC, which is beneficial for $\mathrm{EH}$.
} 
information decoding unit of the $i^{\text {th }}$ receiver equipment is expressed as

$$
y_{D, i}(t)=\left(\mathbf{g}_{i}^{H} \mathbf{x}(t)+n_{R, i}(t)\right)+n_{D, i}(t),
$$

where $n_{D, i}(t)$ is the additional zero-mean Gaussian noise with a variance of $\sigma_{D, i}^{2}$ incurred due to the circuitry and other relevant operations at the ID block of the $i^{\text {th }}$ receiver. For $i^{\text {th }}$ receiver being a part of the $k^{\text {th }}$ multicast group $\mathcal{Z}_{k}$, the signal-to-interference-and-noise ratio (SINR) is given by

$$
\Upsilon_{i}=\frac{\left|\mathbf{w}_{k}^{H} \mathbf{g}_{i}\right|^{2}}{\sum_{\ell \neq k}\left|\mathbf{w}_{\ell}^{H} \mathbf{g}_{i}\right|^{2}+\sigma_{R, i}^{2}+\sigma_{D, i}^{2}}, \forall \ell=\{1, \ldots, \Psi\} .
$$

The signal dedicated for EH block of the $i^{\text {th }}$ receiver is

$$
y_{E, i}(t)=\mathbf{g}_{i}^{H} \mathbf{x}(t)+n_{R, i}(t) .
$$

Therefore, the energy extracted by the EH unit of $i^{\text {th }}$ receiver is given as, $\mathcal{E}_{i}^{\mathcal{L}}=\zeta_{i}\left(\sum_{k=1}^{\Psi}\left|\mathbf{w}_{k} \mathbf{g}_{i}\right|^{2}+\sigma_{R, j}^{2}\right)$, where $0<\zeta_{i} \leq 1$ is the energy conversion efficiency of the $\mathrm{EH}$ unit at the corresponding receiver. Note that $\mathcal{E}_{i}^{\mathcal{L}}$ is theoretically valid in order to represent a linear EH operation, however its practical implementation is questionable. Thus, this calls for the adoption of a non-linear EH model [16]. In this regard, we define the energy harvested at the receiver as follows

$$
\mathcal{E}_{i}^{\mathcal{N}}=\frac{\mathcal{E}^{\prime}}{1-\phi} \cdot\left(\frac{1}{1+e^{\left(-\alpha\left(\sum_{k=1}^{\Psi}\left|\mathbf{w}_{k} \mathbf{g}_{i}\right|^{2}\right)+\alpha \beta\right)}}-\phi\right)
$$

where $\phi \triangleq \frac{1}{1+\exp (\alpha \beta)}$, the constant $\mathcal{E}^{\prime}$ is obtained by determining the maximum harvested energy on the saturation of the energy harvesting circuit, and $\alpha$ and $\beta$ are specific for the capacitor and diode turn-on voltage metrics at the EH circuit. Practically, a standard curve-fitting tool based on analytical data may be used to decide the appropriate values of $\mathcal{E}^{\prime}, \alpha$, and $\beta$. It is interesting to note the hidden linearity aspect within the non-linear EH expression in (5), which can be useful in converting a non-linear EH constraint to a linear form, without loss of generality. The corresponding transformation is provided in (16) of Appendix A. We assume normalized time slots to use the terms power and energy interchangeably.

In the succeeding section, we formulate an optimization problem corresponding to precoder designs for minimization of the total transmit power in the three aforementioned scenarios. Suitable solutions are obtained within polynomial time by employing adequate transformations and relaxations.

\section{TRAnsmit POWER Minimization}

In this section, we formulate the optimization problem corresponding to minimization of the overall transmitted power by the transmitter subjected to minimum SINR and minimum EH constraints at the corresponding users/groups. The overall optimization problem (encapsulating the three aforementioned scenarios) to ensure the co-existence of the three user types in MG-MC precoding scheme can subsequently be written in its mathematical form as follows

$$
\begin{aligned}
&(P 1): \min _{\left\{\mathbf{w}_{k}\right\}_{k=1}^{\Psi}} \sum_{k=1}^{\Psi} \mathbf{w}_{k}^{H} \mathbf{w}_{k} \\
& \text { s.t. } \quad(C 1): \frac{\mathbf{w}_{k}^{H} \mathbf{G}_{i} \mathbf{w}_{k}}{\sum_{\ell \neq k} \mathbf{w}_{\ell}^{H} \mathbf{G}_{i} \mathbf{w}_{\ell}+\sigma_{R, i}^{2}+\sigma_{D, i}^{2}} \geq \gamma_{i},\left\{\begin{aligned}
\psi(a): & \forall i \in \mathcal{Z}_{k}, \forall k \in\{1, \ldots, Z\}, \\
& \forall \ell \in\{1, \ldots, Z+1\} \\
\psi(b): & \forall i \in \mathcal{Z}_{k}, \forall k \in\{1, \ldots, Z\}, \\
& \forall \ell \in\{1, \ldots, Z\} \\
\psi(c): & \forall i, \forall k, \forall \ell \in\{1, \ldots, K\}
\end{aligned}\right. \\
&(C 2): \zeta_{j}\left(\sum_{k=1}^{\Psi} \mathbf{w}_{k}^{H} \mathbf{G}_{j} \mathbf{w}_{k}+\sigma_{R, j}^{2}\right) \geq \xi_{j}^{\prime} \\
& \forall j \in \mathcal{Z}_{Z+1},
\end{aligned}
$$

where $\gamma_{i}$ is the SINR threshold at the $i^{\text {th }}$ user, $\xi_{j}^{\prime}$ is the transformed harvested energy demand ${ }^{3}$ at $j^{\text {th }}$ user (where $i$ can be equal to $j$ for some cases, in general), and $\mathbf{G}_{i}=\mathbf{g}_{i} \mathbf{g}_{i}^{H}$. It is clear that the formulated problem $(P 1)$ is not convex

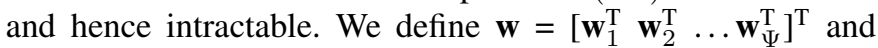
$\mathbf{W}=\mathbf{w} \mathbf{w}^{H}$, with $\mathbf{W}_{k}=\mathbf{w}_{k} \mathbf{w}_{k}^{H}$ indicating the $(k, k)^{\text {th }}$ block of W. With the help of these notations, $(P 1)$ can be represented using semi-definite relaxation (SDR) as follows

$$
\begin{aligned}
& (P 2): \min _{\left\{\mathbf{W}_{k}\right\}_{k=1}^{\Psi}} \sum_{k=1}^{\Psi} \operatorname{Tr}\left\{\mathbf{W}_{k}\right\} \\
& \text { s.t. }(C 1): \operatorname{Tr}\left\{\mathbf{G}_{i} \mathbf{W}_{k}\right\}-\gamma_{i} \sum_{\ell \neq k} \operatorname{Tr}\left\{\mathbf{G}_{i} \mathbf{W}_{\ell}\right\} \\
& \geq \gamma_{i}\left(\sigma_{R, i}^{2}+\sigma_{D, i}^{2}\right), \\
& \left\{\begin{aligned}
\psi(a): & \forall i \in \mathcal{Z}_{k}, \forall k \in\{1, \ldots, Z\}, \\
& \forall \ell \in\{1, \ldots, Z+1\}, \\
\psi(b): & \forall i \in \mathcal{Z}_{k}, \forall k \in\{1, \ldots, Z\}, \\
& \forall \ell \in\{1, \ldots, Z\}, \\
\psi(c): & \forall i, \forall k, \forall \ell \in\{1, \ldots, K\},
\end{aligned}\right. \\
& (C 2): \sum_{k=1}^{\Psi} \operatorname{Tr}\left\{\mathbf{G}_{j} \mathbf{W}_{k}\right\} \geq \frac{\xi_{j}^{\prime}}{\zeta_{j}}-\sigma_{R, j}^{2}, \\
& \forall j \in \mathcal{Z}_{Z+1}, \\
& (C 3): \mathbf{W}_{k} \succcurlyeq 0 \text {. }
\end{aligned}
$$

The SDR transforms the non-convex $(P 1)$ into a convex problem as in $(P 2)$, which can be solved easily with the help of convex programming tool CVX [17]. Let $\mathbf{W}^{\star}$ denote the solution of the relaxed problem in $(P 2)$. Then, $\mathbf{W}^{\star}$ is considered the optimal solution iff $\operatorname{rank}\left(\mathbf{W}_{k}^{\star}\right)=1$ [18]. It was shown in [19] that $\operatorname{arank}\left(\mathbf{W}_{k}^{\star}\right)=2$ is also considered optimal, which can however be reduced to $\operatorname{rank}\left(\mathbf{W}_{k}^{\star}\right)=1$ at an additional computation cost thereby penalizing the system performance and raising a possibility of constraints not being satisfied anymore. Therefore, it is worth mentioning that $\operatorname{rank}\left(\mathbf{W}_{k}^{\star}\right) \leq$ 2 implies that $\mathbf{W}_{k}^{\star}$ is indeed the optimal solution. In other

\footnotetext{
${ }^{3}$ Note that $(\mathrm{C} 2)$ is a linear constraint introduced to simplify the problem. Proof for the corresponding transformation is provided in Appendix A.
} 


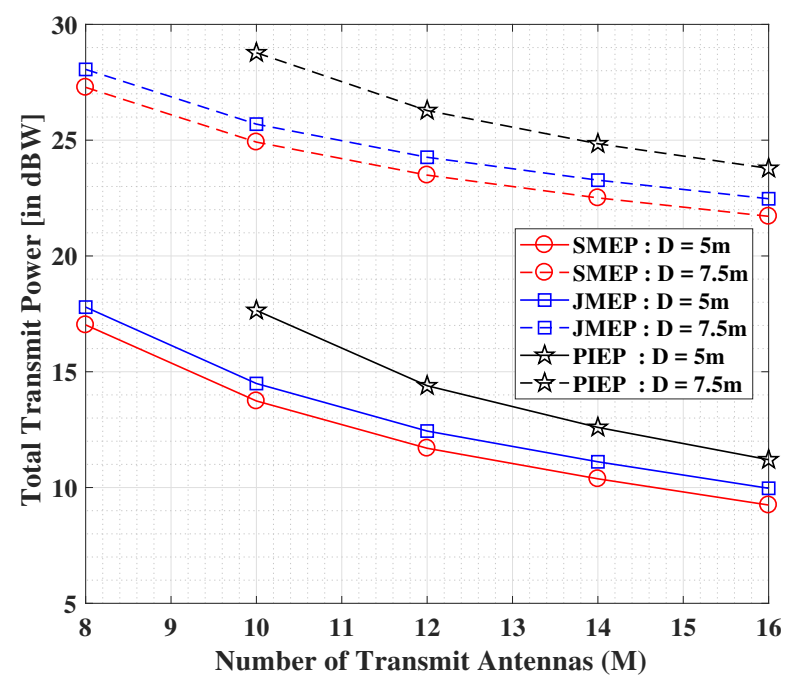

Fig. 4: Performance analysis of SMEP, JMEP and PIEP in terms of total transmit power versus the number of transmit antennas with variation in distance where $\gamma_{i}=5 \mathrm{~dB}$ and $\xi_{i}=1 \mu \mathrm{J}$.

cases, i.e., for $\operatorname{rank}\left(\mathbf{W}_{k}^{\star}\right)>2$, the solution is considered suboptimal with further scope of improvement. The corresponding performance analysis is carried out with the help of numerical results, as discussed in the following section.

\section{Simulation Results}

In this section, we present the performance benefits of the proposed SMEP framework in comparison to the benchmark methods, namely JMEP and PIEP. The three cases viz-a-viz., SMEP, JMEP, and PIEP are implemented using the convex programming tool CVX [17], with solutions obtained via SEDUMI solver. We assume an ITU-R indoor model (2-floor office scenario) to generate channel realizations with the pathloss exponent given by [20]

$$
\text { PL }(\text { in } \mathrm{dB})=20 \log _{10}(F)+N \log _{10}(D)+P_{f}(n)-28 \text {, }
$$

where $F$ is the operational frequency (in $\mathrm{MHz}$ ), $N$ is the distance power loss coefficient, $D$ is the separation distance (in metres) between the transmitter and end-user(s) (with $D$ $>1 \mathrm{~m}), P_{f}(n)=15+4(n-1):$ is the floor penetration loss factor (in $\mathrm{dB}$ ), and $n$ is the number of floors between the transmitter and the end-user(s) (with $n \geq 0$ ). Specifically, the chosen parametric values are : $F=2 \mathrm{GHz}, D=5 \mathrm{~m}$ (unless specified otherwise), $N=30$, and $P_{f}(2)=19 \mathrm{~dB}$. The transmitter is assumed to be equipped with $M=16$ antennas (unless specified otherwise) while $K=10$ users are distributed within $(Z+1)=5$ multicasting groups as follows: $\mathcal{Z}_{1}=\left\{\mathcal{U}_{1}, \mathcal{U}_{3}, \mathcal{U}_{4}\right\}, \mathcal{Z}_{2}=\left\{\mathcal{U}_{2}, \mathcal{U}_{5}\right\}, \mathcal{Z}_{3}=\left\{\mathcal{U}_{6}, \mathcal{U}_{8}\right\}, \mathcal{Z}_{4}=$ $\left\{\mathcal{U}_{7}, \mathcal{U}_{9}, \mathcal{U}_{10}\right\}$, and $\mathcal{Z}_{5}=\left\{\mathcal{U}_{1}, \mathcal{U}_{2}, \mathcal{U}_{4}, \mathcal{U}_{5}, \mathcal{U}_{7}, \mathcal{U}_{8}, \mathcal{U}_{10}\right\}$, where $\mathcal{Z}_{5}$ is the energy harvesting group of users while the remaining $\left(\mathcal{Z}_{1}, \ldots, \mathcal{Z}_{4}\right)$ groups are comprised of information users. We set to $\sigma_{R, i}^{2}=-110 \mathrm{dBW}, \sigma_{D, i}^{2}=-80 \mathrm{dBW}$ and $\zeta_{i}=0.6$. Furthermore, an average of 500 random channel realizations (with random placement of end-users in every realization) is presented for each experiment. The constants corresponding to the non-linear EH circuit are chosen as $\mathcal{E}^{\prime}=2.8 \mathrm{~mJ}, \alpha=$

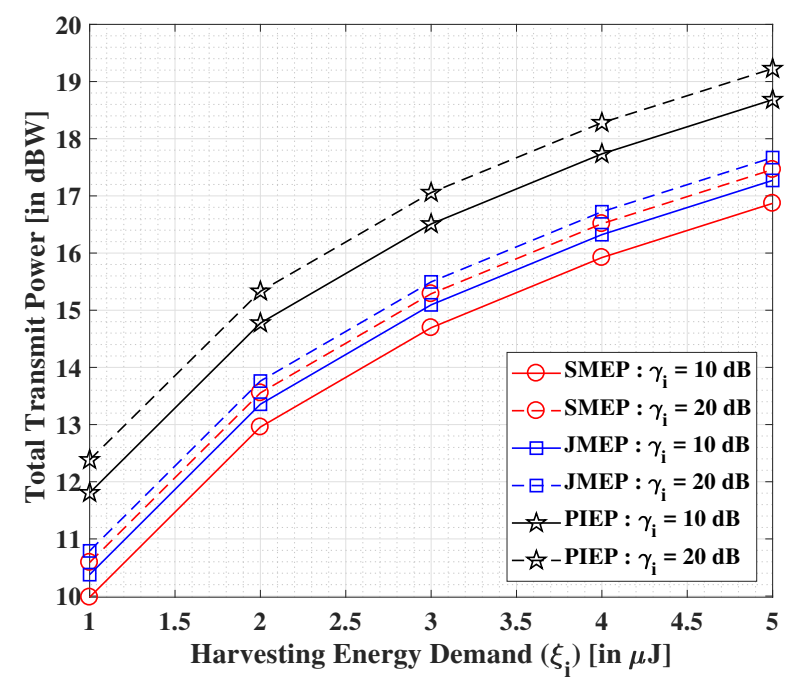

Fig. 5: Performance analysis of SMEP, JMEP and PIEP in terms of total transmit power versus the harvested energy demands with variation in the SINR of users where $D=5 \mathrm{~m}$.

1500 , and $\beta=0.0022$ [16].

It is found that the solutions of both JMEP and PIEP are indeed rank $=1$ for most of the experiments (and some possibilities of rank $=2$ ) whereas such an outcome cannot be assured to be always true, in general. The solution corresponding to SMEP is found to be unit rank for lower values of SINR and harvested energy demands. Further, this aspect is seen to diminish with higher demands of SINR and harvested energy where multi-rank solutions are obtained indicating that the relevant information is conveyed using the designated MC precoder(s) while energy at corresponding user is acquired via dedicated EH-specific and/or more (other) precoder(s) ${ }^{4}$ as well as all other interfering signals from MC precoders.

Fig. 4 shows the variation in total transmit power (in $\mathrm{dBW}$ ) with increasing number of array antennas at the transmitter for $\gamma_{i}=5 \mathrm{~dB}$ and $\xi_{i}=1 \mu \mathrm{J}$. Herein, we compare the proposed SMEP with the two benchmarks JMEP and PIEP, respectively. It is observed that the system performance for all the scenarios improves considerably in terms of the total transmit power with increasing number of transmit array antennas, with SMEP performing appreciably better in comparison to JMEP and PIEP. Furthermore, a same trend is observed when the distance between the transmitter and end-users is increased to $D=$ $7.5 \mathrm{~m}$. However, an expected increase in the total transmit power is also seen in this case. It is also noteworthy that JMEP and SMEP are operational even with lower number of transmit array antennas in comparison to the number of endusers while the former (number of transmit array antennas) should be equal or greater than the latter (number of endusers) to ensure operability of PIEP.

\footnotetext{
${ }^{4}$ Noticeably, a multi-rank solution is obtained for $\left\{\mathbf{W}_{k}\right\}$ corresponding to the EH group, implying that in order to serve EH specific users, more precoders would be required. The ID users are served using the beams of corresponding $Z$ MC precoders while $\mathrm{EH}$ users utilize $\operatorname{rank}\left(\mathbf{W}_{k}\right)$ precoders for collecting energy. Furthermore, the randomization technique [7] may be implemented to reduce the multi-rank solution for $\left\{\mathbf{W}_{k}\right\}$ to a unit rank thereby introducing additional computational penalties.
} 
In Fig. 5, we illustrate the effect on the total transmit power (in $\mathrm{dBW}$ ) with increasing demand of the harvested energy and the SINR threshold. Performance benefits of the proposed SMEP are seen over JMEP and PIEP for both the cases, i.e., increasing the EH demand and increasing the SINR demand at the desired user, respectively. The total transmit power values show an increasing trend with the growing demands of SINR and/or EH thresholds.

The outcomes imply that the solutions for JMEP and PIEP are optimal (for the respective cases individually) while suboptimal results are obtained for SMEP. This means that even with additional precoder(s) in comparison to JMEP, SMEP is seen to provide better performance. It is needless to mention that adoption of separate precoder designs for ID and EH operations does not only reduces the complexity at the transmit source, but also improves the overall system performance. The operation of PIEP involves same number of precoders as the users, which is good for individual users but it naturally imposes an overall high power consumption.

\section{CONCLUSion}

We considered precoding with selective energy harvesting in the context of multi-group multicasting with assumption of three wireless user types that are capable of information decoding only, energy harvesting only, and information decoding and/or energy harvesting, respectively. In this regard, transmit power minimization problem was formulated to optimize the precoders subject to constraints on minimum SINR and EH demands at the corresponding users. Furthermore, this problem is solved and analyzed using the semidefinite relaxation technique under three scenarios, namely, Separate Multicast and Energy Precoding Design (SMEP), Joint Multicast and Energy Precoding Design (JMEP), and Per-User Information and/or Energy Precoding Design (PIEP), respectively. Superior performance of SMEP was shown over JMEP and PIEP, although sub-optimal solution for SMEP and optimal solutions for JMEP and PIEP, respectively, were obtained.

\section{APPENDIX A}

\section{CONVERSION OF NON-LINEAR ENERGY HARVESTING CONSTRAINT TO LINEAR CONSTRAINT}

The non-linear EH constraint at $i^{\text {th }}$ user is given by

$$
\frac{\mathcal{E}^{\prime}}{1-\phi} \cdot\left(\frac{1}{1+e^{\left(-\alpha\left(\sum_{k=1}^{\psi}\left|\mathbf{w}_{k} \mathbf{g}_{i}\right|^{2}\right)+\alpha \beta\right)}}-\phi\right) \geq \xi_{i},
$$

where $\xi_{i}$ is the harvested energy demand at the $i^{\text {th }}$ user.

The expression in (15) can be re-arranged and written as

$$
\frac{\mathcal{E}^{\prime}}{1-\phi} \cdot\left(\frac{1}{1+e^{\left(-\alpha \mathcal{E}_{i}^{\mathcal{L}} / \zeta_{i}+\alpha \sigma_{R, i}^{2}+\alpha \beta\right)}}-\phi\right) \geq \xi_{i} .
$$

Further simplification of (16) leads to the equivalent linear EH constraint

$$
\mathcal{E}_{i}^{\mathcal{L}} \geq \xi_{i}^{\prime}
$$

where

$$
\xi_{i}^{\prime}=\zeta_{i}\left(\sigma_{R, i}^{2}+\beta-\frac{1}{\alpha} \ln \left(\frac{(1-\phi)\left(\mathcal{E}^{\prime}-\xi_{i}\right)}{(1-\phi) \mathcal{E}^{\prime}+\phi \xi_{i}}\right)\right) .
$$

From (18), it is clear that $\xi_{i}^{\prime}$ is an up-scaled version of $\xi_{i}$ and that the constraints in (12) and (15) are equivalent. QED.

\section{ACKNOWLEDGMENT}

This work was partially supported by the Luxembourg National Research Fund (FNR) in the framework of the FNR-FNRS bilateral project "InWIP-NET : Integrated Wireless Information and Power Networks".

\section{REFERENCES}

[1] Q. Shi, L. Liu, W. Xu, and R. Zhang, "Joint Transmit Beamforming and Receive Power Splitting for MISO SWIPT Systems," IEEE Trans. Wireless Commun., vol. 13, no. 6, pp. 3269-3280, June 2014.

[2] S. Gautam and P. Ubaidulla, "Relay Selection and Transceiver Design for Joint Wireless Information and Energy Transfer in Cooperative Networks," in 85th Veh. Tech. Conf. (Spring). IEEE, 2017, pp. 1-5.

[3] R. Tandon, S. A. Jafar, S. Shamai, and H. V. Poor, "On the Synergistic Benefits of Alternating CSIT for the MISO Broadcast Channel," IEEE Trans. Inf. Theory, vol. 59, no. 7, pp. 4106-4128, July 2013.

[4] L. R. Varshney, "Transporting information and energy simultaneously," in IEEE Int. Symp. Inf. Theory, July 2008, pp. 1612-1616.

[5] M. R. A. Khandaker and K. Wong, "SWIPT in MISO Multicasting Systems," IEEE Wireless Commun. Lett., vol. 3, no. 3, pp. 277-280, June 2014.

[6] Z. Ding, C. Zhong, D. W. K. Ng, M. Peng, H. A. Suraweera, R. Schober, and H. V. Poor, "Application of smart antenna technologies in simultaneous wireless information and power transfer," IEEE Comm. Mag., vol. 53, no. 4, pp. 86-93, April 2015.

[7] N. D. Sidiropoulos and T. N. Davidson and, "Transmit beamforming for physical-layer multicasting," IEEE Trans. Signal Process., vol. 54, no. 6, pp. 2239-2251, June 2006.

[8] J. Krivochiza, J. M. Duncan, S. Andrenacci, S. Chatzinotas, and B. Ottersten, "FPGA Acceleration for Computationally Efficient SymbolLevel Precoding in Multi-User Multi-Antenna Communication Systems," IEEE Access, vol. 7, pp. 15509-15520, 2019.

[9] M. Alodeh, S. Chatzinotas, and B. Ottersten, "User Selection for Symbol-Level Multigroup Multicasting Precoding in the Downlink of MISO Channels," in IEEE Int. Conf. Comm. (ICC), May 2018, pp. 1-7.

[10] D. Christopoulos, S. Chatzinotas, and B. Ottersten, "Weighted Fair Multicast Multigroup Beamforming under Per-antenna Power Constraints," IEEE Trans. Signal Process., vol. 62, no. 19, pp. 5132-5142, 2014.

[11] Ö. T. Demir and T. E. Tuncer, "Multi-group multicast beamforming for simultaneous wireless information and power transfer," in 23rd European Sig. Processing Conf. (EUSIPCO), Aug 2015, pp. 1356-1360.

[12] Ö. T. Demir and T. E. Tuncer, "Antenna Selection and Hybrid Beamforming for Simultaneous Wireless Information and Power Transfer in Multi-Group Multicasting Systems," IEEE Trans. Wireless Comm., vol. 15, no. 10, pp. 6948-6962, Oct 2016.

[13] M. Alodeh, D. Spano, A. Kalantari, C. G. Tsinos, D. Christopoulos, S. Chatzinotas, and B. Ottersten, "Symbol-Level and Multicast Precoding for Multiuser Multiantenna Downlink: A State-of-the-Art, Classification, and Challenges," IEEE Commun. Surveys Tuts., vol. 20, no. 3, pp. 1733-1757, thirdquarter 2018.

[14] D. Mishra, G. C. Alexandropoulos, and S. De, "Energy Sustainable IoT With Individual QoS Constraints Through MISO SWIPT Multicasting," IEEE Internet Things J., vol. 5, no. 4, pp. 2856-2867, Aug 2018.

[15] B. Clerckx and E. Bayguzina, "Waveform Design for Wireless Power Transfer," IEEE Trans. Signal Process., vol. 64, no. 23, pp. 6313-6328, 2016.

[16] J. Guo, H. Zhang, and X. Zhu, "Theoretical analysis of RF-DC conversion efficiency for class-F rectifiers," IEEE Trans. Microw. Theory Techn., vol. 62, no. 4, pp. 977-985, 2014.

[17] M. Grant and S. Boyd, "CVX: Matlab software for disciplined convex programming, version 2.1," http://cvxr.com/cvx, Mar. 2014.

[18] M. Bengtsson and B. Ottersten, "Optimum and Suboptimum Transmit Beamforming," in Handbook of Antennas in Wireless Communications. CRC press, 2001

[19] R. Madani, G. Fazelnia, and J. Lavaei, "Rank-2 matrix solution for semidefinite relaxations of arbitrary polynomial optimization problems," constraints, vol. 21, pp. 25, 2014.

[20] P. Series, "Propagation data and prediction methods for the planning of indoor radiocommunication systems and radio local area networks in the frequency range $900 \mathrm{MHz}$ to $100 \mathrm{GHz}$," Recommendation ITU-R, pp. 1238-7, 2012. 